\title{
Family history-taking in community family practice: Implications for genetic screening
}

Louise S. Acheson, MD, MS, Georgia L. Wiesner, MD, Stephen J. Zyzanski, PhD, Meredith A. Goodwin, MA, and Kurt C. Stange, $M D, P h D$

\begin{abstract}
Purpose: To identify characteristics of physicians, patients, and visits associated with obtaining family history information in community family practice. Methods: Research nurses directly observed 4454 patient visits to 138 family physicians and reviewed office medical records. Results: Family history was discussed during $51 \%$ of visits by new patients and $22 \%$ of visits by established patients. Physicians' rates of family history-taking varied from $0 \%$ to $81 \%$ of visits. Family history was more often discussed at well care rather than illness visits. The average duration of family history discussions was $<2.5$ minutes. Conclusions: These data can form the basis for realistic interventions to increase the use of family history in primary care. Genetics in Medicine, 2000:2(3):180-185.
\end{abstract}

Key Words: Family history, primary care, family practice, genetic services

Family physicians are in a central position to identify families and individuals at risk for inherited health problems. Some of these patients may benefit from specialized genetic services or from preventive care tailored according to their familial risks of disease (e.g., colon cancer screening). ${ }^{1}$ The primary tool for initial genetic assessment is the family history, but there are few data on family history-taking in primary care. Most family practice training programs teach residents how to record pedigrees and to be alert to family information that could have a bearing on patients' health, although few have included genetic diagnosis and counseling in the core curriculum. ${ }^{2,3}$ By self-report, $95 \%$ of primary care physicians surveyed in the Pacific Northwest "take a family history as part of routine care."4

However, using direct observation of physicians with patients, our colleagues have shown that some family history information is discussed at only half $(51 \%)$ of visits by new patients and $22 \%$ of visits by established patients to their family physicians. ${ }^{5}$ Approximately $40 \%$ of family physicians' patients have information recorded in the office record about a family history (positive or negative) of breast or colon cancer or alcoholism. A pedigree diagram is present in the medical record of $11 \%$ of patients. These observations suggest that the potential for family physicians to recognize their patients' genetic risks through obtaining a family history is being underutilized. Higher rates of family history-taking will be desirable as the assessment of genetic predispositions becomes a more powerful tool for guiding many aspects of medical care. ${ }^{6}$

Therefore, we studied the characteristics of patients, office visits, and family physicians that were associated with discus-

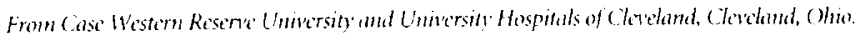
Lonise Achesen, MD, MS, Departunen of Family Medicine, Casc Westem Reseme Universitv.

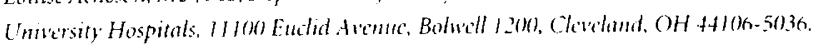

Recined: February 7, 2000.

Acceptit: March 24, 2000 sion of the family history during directly observed patientphysician encounters. Our aim was to elucidate factors associated with recording information about a patient's family history. The resulting insights would be useful to design interventions that might increase family history-taking for patients seeing family physicians.

Primary care involves continual prioritization of clinical tasks, differing in value for each patient, that compete for scarce provider time. ${ }^{7}$ We hypothesized that family physicians might use patient characteristics, such as age, and brief, nonspecific "screening" questions about family history to select some patients whose family history would be pursued in greater detail because of a perception of increased familial risk or an expectation of clinical benefits from this information. ${ }^{8}$ However, because genetic assessment is likely to be viewed as part of preventive care, rather than immediately applicable to the care of acute illnesses that comprise the bulk of family practice visits, we hypothesized that most discussions of family history would occur in the context of well adult, well child, or prenatal care.

\section{METHODS}

\section{Sample and data collection}

The methods of the Direct Observation of Primary Care study have been described in detail previously..$^{9,10}$ This study of the content and context of primary care practice enrolled 138 community family physicians in 84 northeastern Ohio practices, who had volunteered to participate in a study of the content of family practice. In order to minimize a Hawthorne effect, no specific hypotheses were shared with study participants beforehand.

Research nurses observed each physician on 2 separate days, collecting data through direct observation of patient visits, review of medical records and billing data, patient exit questionnaires, physician questionnaires, a practice environment 
checklist, and field notes. The way in which time was spent during each visit was measured using the modified" Davis Observation Code (DOC). ${ }^{9}$ The DOC is a checklist of 20 different types of activity (e.g., "history-taking," "physical examination," "discussing family issues," etc.) that were assessed by the research nurse during each 20 -second interval of the doctor-patient encounter. At the time of each visit, the observing nurse also completed a checklist noting the reason for the visit, whether any family history was elicited, whether another family member was present, and whether another family member's problem was discussed during the visit.

Subsequent medical record review recorded whether family history was documented in the record for the observed visit, and (in the second half of the study only) whether specific family history items (a positive or negative family history of breast cancer, colon cancer, or alcoholism, and a family tree diagram) were present anywhere in the office medical record. The medical record was also used to determine whether the patient was an established or new patient of the practice. Information on physician demographics and practice characteristics was obtained by self-report from $128(93 \%)$ of the study physicians via a physician questionnaire.

The patient sample consisted of consecutive patients seen by each study physician during 2 days of observation, approximately 4 months apart. Patients were informed about the study in the waiting room prior to seeing the physician, and were enrolled if they gave verbal informed consent. This research protocol was approved by the Institutional Review Board for Human Investigation of University Hospitals of Cleveland.

\section{Analyses}

The main outcome variable for this analysis was whether any family history was discussed during the observed office visit. Descriptive statistics were calculated with patients and physicians as the units of analysis. The patient sample was stratified by whether patients were new or established, because gathering of family history information is likely to be different for patients who are not known to the physician. Forty-one patients with missing data regarding their status as new or established patients were excluded from analysis. Analyses of physician characteristics and rates of family history-taking were adjusted for the proportion of well care visits seen by each physician, using an analysis of covariance. Comparisons of visits with and without family history-taking were made by t-tests for continuous variables and chi-square statistics for categorical variables.

Certain independent variables (whether the patient was accompanied by another family member, type of insurance, the number of problems addressed, and length of the visit) were associated with the patient's age. The number of problems addressed and the length of the visit were also associated with the reason for visit. Therefore, analyses of family history-taking in terms of these variables were performed both with and without adjusting for age and reason for visit. Because adjusting for age and reason for visit did not change the results, only the unadjusted comparisons are reported here.

\section{RESULTS}

Family physicians participating in this study were similar in demographics and spectrum of practice to the national practicing physician membership of the American Academy of Family Physicians (AAFP), ${ }^{9,12}$ except that, in keeping with recent trends, a higher proportion of physicians in the study sample were female ( $27 \%$ vs. $21 \%$ in AAFP) and residencytrained ( $89 \%$ vs. $73 \%$ in AAFP). In keeping with regional practice patterns, fewer of the study physicians provided obstetrical care than among AAFP physician members nationwide: in $1995,21 \%$ of study physicians delivered babies versus $31 \%$ of AAFP members. ${ }^{12}$ The study physicians had practiced at their current site for a mean of $10.5 \pm 7.8$ years.

Among patients seen on observation days, $89 \%$ participated. Participants were slightly older than nonparticipants, but similar in gender and race. Reasons most commonly given for nonparticipation were concern about privacy, embarrassment or shyness, and not wanting an observer present during a gynecologic examination. ${ }^{10}$

Some family history information was discussed during $24.3 \%$ of 4,454 directly observed visits of patients to family physicians in this study. Family physicians varied substantially in how frequently they elicited family histories, ranging from $0 \%$ to $81 \%$ of observed patient visits (Fig. 1). Physicians with fewer years at the current practice site were more likely to elicit a family history from established patients during the observed visits (Table 1). For established patients, there was a trend toward a higher rate of family history-taking by younger, residency-trained physicians. These associations were present after adjustment for the proportion of well care visits to each physician.

Table 2 shows the association of patient characteristics with a family history being discussed during the visit. Overall, $8.6 \%$ of the patients were new to the practice; approximately half

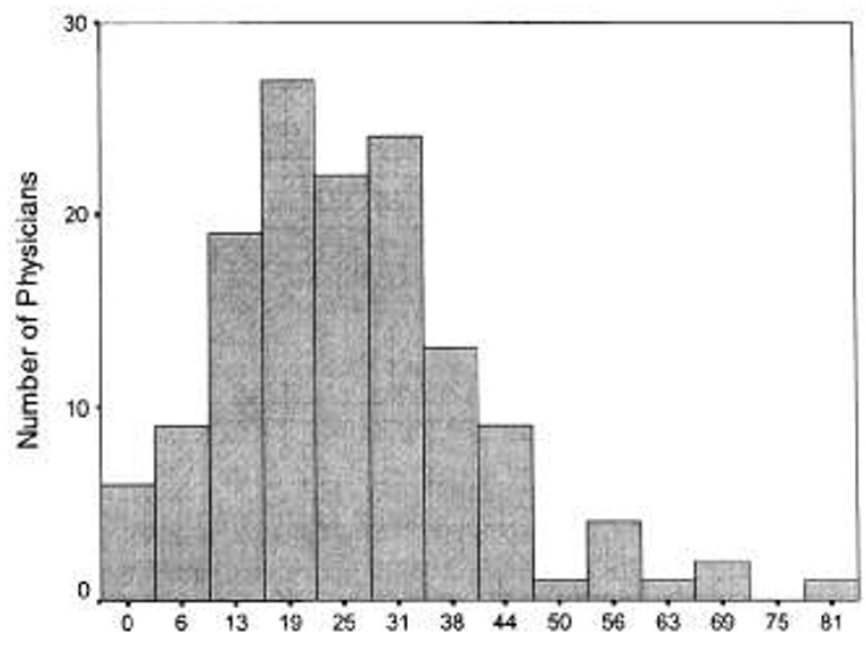

Percent of visits in which a family history was discussed

Fig. 1 Rates of family history-taking for physicians in the Direct Observation of Primary Care study. 
Table 1

Physician characteristics vs. percent of visits in which family history was discussed*

\begin{tabular}{|c|c|c|c|c|}
\hline \multirow[b]{3}{*}{ Physician } & \multicolumn{4}{|c|}{ Percent of visits in which family history discussed } \\
\hline & \multicolumn{2}{|c|}{ New Patient Visits } & \multicolumn{2}{|c|}{ Established Patient Visits } \\
\hline & Correlation & $P$ & Correlation & $P$ \\
\hline Age & -0.01 & 0.93 & -0.17 & 0.06 \\
\hline \multirow[t]{2}{*}{ Years at current practice site } & -0.10 & 0.34 & -0.22 & 0.02 \\
\hline & $\begin{array}{l}\text { Mean } \\
\text { Percent }\end{array}$ & $P$ & $\begin{array}{c}\text { Mean } \\
\text { percent }\end{array}$ & $P$ \\
\hline \multicolumn{5}{|l|}{ Sex } \\
\hline Male $(\mathrm{n}=92)$ & $56.1 \%$ & $0.91+$ & $22.4 \%$ & $0.09+$ \\
\hline Female $(n=36)$ & $55.1 \%$ & & $27.0 \%$ & \\
\hline \multicolumn{5}{|l|}{ Residency trained } \\
\hline Yes $(n=114)$ & $55.9 \%$ & $0.30+$ & $23.7 \%$ & $0.06+$ \\
\hline No $(n=14)$ & $43.2 \%$ & & $16.5 \%$ & \\
\hline \multicolumn{5}{|l|}{ Performs prenatal care } \\
\hline Yes $(n=43)$ & $51.3 \%$ & $0.46+$ & $24.3 \%$ & $0.57+$ \\
\hline No $(n=85)$ & $57.3 \%$ & & $22.9 \%$ & \\
\hline
\end{tabular}

*Analyses adjusted for the percent of well care visits.

+ Probability determined by $\mathrm{F}$ test.

Table 2

Association of patient characteristics with family history discussed during visit

\begin{tabular}{|c|c|c|c|c|c|c|}
\hline & \multicolumn{3}{|c|}{ New Patients Family History } & \multicolumn{3}{|c|}{ Established Patients Family History } \\
\hline & Yes & No & $P$ & Yes & No & $P$ \\
\hline & $(\mathrm{n}=195)$ & $(n=186)$ & & $(\mathrm{n}=887)$ & $(\mathrm{n}=3186)$ & \\
\hline \multicolumn{7}{|l|}{ Age $^{*}$} \\
\hline$\leq 1$ & 47.4 & 52.6 & 0.03 & 17.3 & 82.7 & $<0.001$ \\
\hline $2-14$ & 47.9 & 52.1 & & 20.6 & 79.4 & \\
\hline $15-44$ & 50.0 & 50.0 & & 24.8 & 75.2 & \\
\hline $45-64$ & 66.2 & 33.8 & & 23.3 & 76.7 & \\
\hline$\geq 65$ & 33.3 & 66.7 & & 17.6 & 82.4 & \\
\hline Sex (\% female) & 52.8 & 57.0 & 0.41 & 66.0 & 61.2 & + \\
\hline Health status $(1=$ poor, $5=$ excellent $)$ & 4.0 & 3.9 & 0.48 & 3.8 & 3.7 & 0.04 \\
\hline Marital status $++(\%$ married $)$ & 61.5 & 45.1 & 0.006 & 66.1 & 62.0 & 0.05 \\
\hline Number of years as patient of physician & NA & NA & & 5.6 & 5.9 & 0.22 \\
\hline Number of visits to observed doctor in past year & NA & NA & & 4.1 & 4.8 & $<0.001$ \\
\hline
\end{tabular}

*Percent with family history in each age category.

$+P=0.02$ for well care visits; $P=0.08$ for illness.

++ Only applicable to patients 18 and older.

(51\%) of new patients discussed family history with the physician at their first visit. Family history was discussed in $22 \%$ of established patients' visits. Patients 65 years or older were least likely to be asked about family history at their visits. Patients with Medicare insurance were less likely to be involved in family history-taking; there was no other association of type of insurance with family history-taking (data not shown). At the initial visit, physicians were more likely to ask married adults than single adults for family history information. Established patients had been patients of their physician for a mean of 5.7 years; the duration of this relationship was not associated with history-taking at the observed visit, but patients who had seen their physician more often during the past year were less likely to be asked about family history at the observed visit. Stratify- 
ing the analysis of patient characteristics according to the type of visit (i.e., well care vs. illness visits), did not change the associations shown in Table 2, except for gender. Among established patients, women coming for well care were more likely to discuss family history than men, with a comparable but not statistically significant trend for women coming for illness visits. The patient's health status or educational attainment were not associated with family history-taking.

Table 3 shows characteristics of outpatient visits in which the patient's family history was addressed. These visits were, on the average, 3 minutes longer ( 12.3 vs. 9.1 minutes for established patients) than visits in which no family history was elicited. Furthermore, a higher percentage of the time intervals during these visits was spent discussing family issues ( $8.3 \%$ vs. $15 \%$ of intervals for established patients), and a slightly but significantly larger number of health problems were addressed. Patients who were accompanied by another family member were less likely to discuss family history. Family history was much more likely to be obtained during visits for well person care, especially for established patients. Well care visits made up $12 \%$ of all visits observed; visits for acute illness $58 \%$; chronic illness $24 \% .^{9}$

We hypothesized that once family history information had been recorded in the medical record, discussion of the family history might be less likely during subsequent visits. Therefore, the data were analyzed to determine what proportion of patients, in whose visits no family history-taking was observed, had family history information in the office medical record. Data on three items (presence in the record of information about a positive or negative family history of breast cancer or colon cancer, and presence of a pedigree diagram) were available only for the second observed practice day of each physician, for 191 new and 2152 established patient visits. Thirtyseven percent of established patients who did not discuss family history during the observed visit did have information about a family history of colon or breast cancer in their medical records. Twenty-four percent of new patients who did not discuss family history during their visit with the physician nevertheless had information in their medical records about a family history of breast and colon cancer. This was contained in questionnaires that new patients were asked to complete before seeing the physician. In contrast, only $4 \%$ of new patients who did not discuss family history had a pedigree diagram in their medical records, compared with $24 \%$ of those whose physicians elicited family history information during the visit. A genetic history was documented in the medical records of $66 \%$ of the 59 pregnant patients whose office visits were observed.

\section{DISCUSSION}

As knowledge of genetic contributions to illness and health grows, the family history is becoming an increasingly important and powerful clinical tool. The Direct Observation of Primary Care study provides information not previously available about the circumstances in which family physicians, the largest group of primary care clinicians in the U.S., elicit family history information during the care of patients in the office.

The findings from this study must be interpreted in the context of its strengths and limitations. The unique value of these data lies in their derivation from multimethod, direct observations of patient care in a variety of community family practices. While the study physicians appear fairly representative of family practitioners nationwide, they were all practicing in Northeastern Ohio; thus the study does not have the potential to capture regional differences in practice patterns. Nevertheless, large geographic differences in family history-taking are unlikely. The National Ambulatory Medical Care Survey revealed that the average office visit to an internist is only 3 minutes longer than visits to family physicians, suggesting that similar time limitations and competing demands will be operative for primary care practitioners in general. ${ }^{13}$

Table 3

Association of visit characteristics with family history discussed during visit

\begin{tabular}{|c|c|c|c|c|c|c|}
\hline & \multicolumn{3}{|c|}{ New Patients Family History } & \multicolumn{3}{|c|}{ Established Patients Family History } \\
\hline & Yes & No & $P$ & Yes & No & $P$ \\
\hline Length of visit (minutes) & 15.1 & 9.7 & $<0.001$ & 12.3 & 9.1 & $<0.001$ \\
\hline $\begin{array}{l}\text { Proportion of time spent discussing family issues } \\
\text { (\% of intervals) }\end{array}$ & 16.9 & 10.1 & $<0.001$ & 15.0 & 8.3 & $<0.001$ \\
\hline Number of problems addressed & 2.1 & 1.6 & $<0.001$ & 2.0 & 1.8 & $<0.001$ \\
\hline Other family member present (\% yes) & 31.1 & 32.2 & 0.81 & 26.7 & 33.2 & $<0.001$ \\
\hline \multicolumn{7}{|l|}{ Reason for visit $(\%)^{*}$} \\
\hline Acute illness & 44.3 & 55.7 & 0.002 & 18.1 & 81.9 & $<0.001$ \\
\hline Chronic illness & 48.0 & 52.0 & & 19.5 & 80.5 & \\
\hline Well care & 60.7 & 39.3 & & 44.1 & 55.9 & \\
\hline Other & 73.0 & 27.0 & & 25.4 & 74.6 & \\
\hline
\end{tabular}

*Proportion with family history according to reason for visit. 
Because the Davis Observation Code records "discussion of family issues" if any portion of a 15-second observation interval was spent on discussion of a broad range of family topics, family history-taking reported here represents an upper-limit estimate of the time spent discussing the family medical history. This study did not collect further descriptive data on the content or scope of family history information elicited. Likewise, the family tree diagram or "genogram" recorded in some patients' records was not analyzed for its completeness or for the amount of information it contained that would be pertinent to defining familial risks of diseases with a heritable component. In the past, family practitioners who advocated for use of the family genogram in patient care tended to emphasize the analysis of family structure and relationships as much as its use in recognizing patterns of inherited susceptibility to disease. ${ }^{2,14-17}$ Undoubtedly, a process of refocusing the information contained in a "genogram" will occur as more of the genetic contributions to health becomes known. ${ }^{18}$

The study findings have a number of implications for medical care. The wide physician-to-physician variability in rates of family history-taking implies the possibility that a more standardized and consistent approach to collecting basic family history information could enhance patient care. Higher rates of family history-taking were associated with fewer years of practice in the current site. This finding was not due to a higher proportion of new patients or well care visits to younger practices, but may reflect more recent training, both in predoctoral curricula that include genetics and in family practice residencies that emphasize care of patients in the context of the family. This observation is consistent with those of Hofman et al. ${ }^{19}$ who found in a 1991 survey that primary care physicians graduated from medical school after 1970 had better knowledge of genetics than earlier graduates, and Hayflick et al. ${ }^{4}$ who found that primary care physicians with greater knowledge of genetics were more likely to perform appropriate genetic assessments of their patients. Medalie and colleagues ${ }^{20}$ showed that those family physicians in the Direct Observation of Primary Care study who were more likely to discuss and record family history information and to counsel their patients about familial or genetic disease also had a higher rate of preventive service delivery than family physicians with a different practice style; conversely, however, fewer of their patients felt that the physician had in-depth knowledge of them and their families. In order not to compromise other important aspects of primary care, any method for standardizing the collection of family history information should be accomplished in a way that allows individualization of its use within different physician styles and patient needs.

New patient visits are prime opportunities for collecting family history information. Family history was often discussed with new patients even if they initially presented for care of an illness. Additional patients provided family history information through written questionnaires that became part of their medical records. Systems to enhance the collection of family history data before the first office visit would appear to fit well with current practice among family physicians. Family history records that are portable could further contribute to efficiency, given that one quarter of patients in the Direct Observation of Primary Care study had been forced by a change in insurance coverage to find a new physician within the past two years. ${ }^{21}$

Conversely, physicians in a continuity relationship with established patients should consider incorporating into their practices procedures for periodic family history updates. The family history is most likely to be discussed when patients visit the family physician for well care. The longer time scheduled for well care visits and their focus on prevention encourage exploration of the family medical history as an important context for care. However, routines encompassing more than well care visits, if feasible, would be desirable because well care represents only $12 \%$ of outpatient visits ${ }^{9}$ and many adult patients do not visit doctors for well care. ${ }^{22,23}$

Elderly patients represent an untapped resource for family history. The finding that they were substantially less likely than others to be asked about their family histories may represent the need for physicians to attend to multiple chronic medical conditions and changes in functional status during their visits with older patients. In addition, patients and physicians may perceive that family history is less predictive for the personal health of someone who has reached older age. However, the older person is likely to know information about the health of family members of more than one generation, which may be important for assessing the familial risk of illness for younger family members. ${ }^{24}$

In current practice, having another family member present, as occurred in $32 \%$ of observed visits, decreased the likelihood that family history would be discussed. Interventions to increase the use of family history in primary care should consider how to capture the opportunity provided by such visits to engage the family in contributing family history information and in understanding its implications.

According to the "competing demands" model,7 primary care clinicians use sophisticated, but not explicit, clinical judgements to prioritize many potentially important clinical and interpersonal tasks that compete for the limited time with the patient during each encounter. The average time that patients spent face-to-face with the physician was 10 minutes. ${ }^{9}$ In visits that included family history-taking, a substantial proportion $(15 \%)$ of the time intervals included discussion of family issues, but the absolute time was short, amounting to a mean of $<110$ seconds for established patients and $<153$ seconds for new patients. The visits in which family history information was obtained were 3 minutes longer than when no family history was discussed. A similar increase in visit duration (estimated by the physician) had been found previously by Rogers and Rohrbaugh in a randomized, controlled trial of providing prerecorded genograms in family practice. ${ }^{16}$

Given the limited time available, it is understandable that genograms are uncommonly made, despite the importance and usefulness often attributed to them in family practice training and theory. ${ }^{14,15,25}$ Rogers and Durkin ${ }^{26}$ reported that initial construction of a 3-generation family tree for family practice office patients by means of a semistructured interview 
took $15-20$ minutes. In the current study, $11 \%$ of patients' records did include a family tree. Apparently, clinicians sometimes chose to spend the time necessary during initial clinical encounters to construct the genogram, or, since patients had a mean of 4 visits per year, ${ }^{9}$ the family tree may have been built incrementally during more than one encounter.

How and for what purposes will family histories be obtained and interpreted by primary care physicians amid the competing demands to provide care for a wide range of acute and chronic illness, mental health, and preventive service needs? The brief family history obtained in primary care is not comparable in either purpose or scope to the systematic and comprehensive family medical history obtained during genetic consultations. Except during prenatal care, the topics included in a family medical history for an unselected patient are not standardized. ${ }^{27}$ Little is known about the optimal content of a less comprehensive, "screening" family history for various clinical purposes. ${ }^{8}$

The challenge, however, in the era of "genomic medicine," will be for primary care practitioners to develop efficient and systematic ways of obtaining and using family history information for medical care. ${ }^{3,6,28}$ To accomplish this, data collection outside the time frame of an office visit would appear to be necessary. It has been demonstrated that unselected family practice patients can use a workbook or computer program to record their own family trees and family medical histories. ${ }^{16,29,30}$ These tended to be more complete than the family trees recorded by family physicians during the time allotted for patient care. ${ }^{16}$ Computer-assisted telephone interviews, especially for patients without access to personal computers, can be adapted for this purpose ${ }^{31}$ (also see Acheson et al., unpublished data). Protocols to support nongeneticists to act on family history information need to be developed and tested. The combination of a family tree diagram and a screening family history on all primary care patients could help to identify families that might benefit from genetic consultation, counseling, and testing. Because more than $24 \%$ of outpatient visits are to family physicians, and nearly $60 \%$ are to primary care physicians, efforts to increase the feasibility of widespread family history-taking are an important step in introducing patients to the genomic age. ${ }^{32}$

\section{Acknowledgments}

This work was supported by grants from the National Cancer Institute (1RO1 CA 60,862 and 2RO1 CA 60,862) and a Translational Oncology Research Training grant (K12 CA 76,917), by a HRSA Establishment of Departments of Family Medicine grant, by a Family Practice Research Center grant from the American Academy of Family Physicians, and by a Robert Wood Johnson Generalist Physician Faculty Scholar Award to Dr. Stange. The authors are grateful to the physician members of the Research Association of Practicing Physicians (RAPP) and to the office staffs and patients without whose participation this study would not have been possible.

\section{References}

1. Whittemore AS. Genetically tailored preventive strategies: An effective plan for the twenty-first century? Cancer Epidemiol Biomarkers Prev 1999;8:649-658.
2. Rogers JC. Can physicians use family genogram information to identify patients at risk of anxiety or depression? Arch Fam Med 1994;3:1093-1098.

3. Doukas DI. Primary care and the human genome project. Into the breach. Arch Fam Med 1993;2:1179-1183.

4. Hayflick SI, Eiff P, Carpenter L, Steinberger J. Primary care physicians' utilization and perceptions of genetics services. Genet Med 1998;1:13-21.

5. Medalie $\mathrm{IH}$, Zyzanski SI, Langa $\mathrm{D}$, Stange $\mathrm{KC}$. The family in family practice: Is it a reality? J Fam Pract 1998;46:390-396.

6. Pyeritz RE. Family history and genetic risk factors: Forward to the future. JAMA 1997;278:1284-1285.

7. Jaen CR, Stange KC, Nutting P. The competing demands of primary care: A model for the delivery of clinical preventive services. J Fam Pract 1994;38:166-171.

8. Burke W, Pinsky L, Berg A, Press N. Translating genetics into the language of primary care. (Submitted for publication, 1999.)

9. Stange KC, Zyzanski SJ, Jaén CR, Callahan EJ, Kelly RB, Gillanders WR, Shank JC, Chao J, Medalie JH, Miller WL, Crabtree BF, Flocke SA, Gilchrist VI, Langa DM, Goodwin MA. Illuminating the 'black box.' A description of 4454 patient visits to 138 family physicians. J Fam Pract 1998;46:377-389.

10. Stange KC, Zyzanski SI, Smith TF, Kelly R, Langa DM, Flocke SA, Jaén CR. How valid are medical records and patient questionnaires for physician profiling and health services research? A comparison with direct observation of patient visits. Med Care 1998;36:851-867.

11. Callahan EJ, Bertakis KD. Development and validation of the Davis Observation Code. Fam Med 1991;23:19-24.

12. American Academy of Family Physicians. Facts about family practice. Kansas City, MO: American Academy of Family Physicians, 1996.

13. Stafford RS, Saglam D, Causino N, Starfield B, Culpepper L, Marder WD, Blumenthal D. Trends in adult visits to primary care physicians in the United States. Arch Fam Med 1999;8:26-32.

14. Like RC, Rogers J, McGoldrick M. Reading and interpreting genograms: A systematic approach. J Fam Pract 1988;26:407-412.

15. McGoldrick M, Gerson R. Genograms in family assessment. New York: Norton, 1985.

16. Rogers IC, Rohrbaugh M. The SAGE-PAGE trial: Do family genograms make a difference? J Am Board Fam Pract 1991;4:319-326.

17. Troncale JA. The genogram as an aid to diagnosis of distal renal tubular acidosis. J Fam Pract 1983;17:707-708.

18. Daly M, Farmer J, Harrop Stein C, Montgomery S, Itzen M, Costalas JW, Rogatko A, Miller S, Balshem A, Gillespie D. Exploring family relationships in cancer risk counseling using the genogram. Cancer Epidemiol Biomarkers Prev 1999;8:393-398.

19. Hofman KJ, Tambor ES, Chase GA, Geller G, Faden RR, Holtzman NA. Physicians' knowledge of genetics and genetic tests. Acad Med 1993;68:625-632.

20. Medalie J, Zyzanski S, Goodwin M, Stange K. Two physician styles of focusing on the family: Their relation to patient outcomes and process of care. J Fam Pract 2000;49: 209-215.

21. Flocke SA, Stange KC, Zyzanski SI. The impact of insurance type and forced discontinuity on the delivery of primary care. J Fam Pract 1997;45:129-135.

22. Canadian Task Force on the Periodic Health Examination. The Periodic Health Examination 1984: A report of the Periodic Health Examination Task Force. Ot tawa: Health Services Directorate, Health Services and Promotion Branch, Department of National Helath and Welfare, 1984

23. Luckmann R, Melville SK. Periodic health evaluation of adults: A survey of family physicians. J Fam Pract 1995;40:547-554.

24. Bannerman C. The genogram and elderly patients [Letter]. I Fam Pract 1986;23: 426,428 .

25. North S, Marvel K, Hendricks B, Morphew P, North D. Physicians' usefulness ratings of family-oriented clinical tools. J Fam Pract 1993;37:30-34.

26. Rogers J, Durkin M. The semi-structured genogram interview: I. Protocol, II. Evaluation. Fam Sys Med 1984;2:176-188.

27. Van McCrary S, Allen B, Moseley R, Crandall LA, Ostrer H, Curry RW, Dewar MA Nye D. Ethical and practical implications of the human genome initiative for family medicine. Arch Fam Med 1993;2:1158-1163.

28. Collins F. Preparing health professionals for the genetic revolution. IAMA 1997;278: $1285-1286$.

29. Ebell $\mathrm{MH}$, Heaton CJ. Development and evaluation of a computer genogram. J Fam Pract 1988;27:536-538.

30. Gerson R, McGoldrick M. The computerized genogram. Prim Care 1985;12:535-545.

31. Kadison P, Pelletier EM, Mounib EL, Oppedisano P, Poteat HT. Improved screening for breast cancer associated with a telephone-based risk assessment. Prev Med 1998; 27:493-501

32. Woodwell DA. Advance data: National Ambulatory Medical Care Survey, 1996 summary. Atlanta: Centers for Disease Control and Prevention/National Center for Health Statistics, 1997 